\title{
The Rejection of Magnetic Noise From The Wire Using Independent Component Analysis for Magnetocardiogram
}

\author{
K. Kobayashi ${ }^{1}$, Y. Uchikawa ${ }^{2}$, Member, IEEE, T. Simizu ${ }^{1}, \mathrm{~K}$ Nakai $^{3}$, and M. Yoshizawa ${ }^{1}$ \\ ${ }^{1}$ Faculty of Engineering, Iwate University, Iwate 020-8551, Japan \\ ${ }^{2}$ Faculty of Science and Engineering, Tokyo Denki University, Saitama 350-0394, Japan \\ ${ }^{3}$ Laboratory of Medicine, Iwate Medical University, Iwate 020-8505, Japan
}

In magnetocardiogram (MCG) measurements, the magnetic noise from the wire used to suture the sternum after heart surgery becomes a problem. As one method of noise rejection, independent component analysis is effective.

In this study, MCG measurements were carried out under the conditions that the wire was attached to a normal subject and that the wire was not attached. Signal processing by independent component analysis, digital high-pass filter and singer value decomposition analysis were carried out in order to reduce the effect of magnetic noise from the wire. From the comparison of the waveforms after this signal processing and the waveforms where the wire was not attached, it was apparent that the magnetic noise from the wire was reduced by independent component analysis.

From this result, it is clear that independent component analysis is effective for the removal of the magnetic noise from the wire.

Index Terms-High-pass filter, independent component analysis, magnetocardiogram, signal processing, wire noise.

\section{INTRODUCTION}

$\mathbf{R}$ ECENTLY, the clinical application of magnetocardiograms (MCGs) has been widely studied, and analysis of the conduction process [1], [2] and evaluation of ischemic heart disease [3], [4] have been carried out. However, postoperative diagnosis using an MCG becomes difficult in the patient who has a wire fixed in the sternum by the thoracotomy operation, since the magnetic noise from the wire is very great.

As a signal analysis technique, independent component analysis (ICA) is a useful method for separating the independent signals from overlapping signals. ICA is often used by the analysis of magnetoencephalogram (MEG) and electroencephalogram (EEG).

We carried out MCG measurements under the conditions that a wire was attached to a normal subject and that a wire was not attached. The signal processing by ICA was carried out in order to reduce the effect of the magnetic noise from the wire. For the comparison, the signal processing by digital filter (highpass filter: HPF) and singer value decomposition analysis (SVD) were also carried out. We compared the waveforms after this signal processing and the waveforms where the wire was not attached using the correlation coefficient.

\section{METHODS}

The MCGs were measured at 64 points at $2.5 \mathrm{~cm}$ intervals on the chest wall of a normal subject. To simulate of the magnetic noise from the wire in a postoperative, the wire (SUS304) for the operation was placed on the chest wall $(3 \mathrm{~cm}$ under the episternum) of a normal subject. ECG (lead II) was also measured. Fig. 1 shows the 64 measurement positions (fixed circle) on the

Digital Object Identifier 10.1109/TMAG.2005.854851

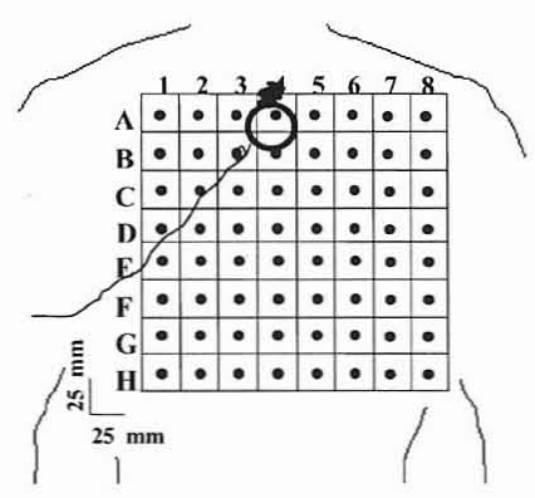

Fig. 1. Measurement 64 positions on body surface and the wire position.

body surface and the wire position (open circle). This wire was equipped with a strap and was fixed by tape to allow simple detachment.

First, the MCG measurements were carried out with the wire attached. Next, the strap was pulled, the wire removed, and MCGs were measured without moving the subject. These measurements differ only in the effect of the wire. This method allows the comparison of the waveforms with the wire attached and those without the wire.

The magnetic field data was processed with a $50 \mathrm{~Hz}$ notch filter and $0.1-90 \mathrm{~Hz}$ band pass filter, and the data was separated into seven components by principal component analysis (PCA). Independent component analysis was done for these data, and it was separated to seven independent components [5], [6]. For each component, a Fourier transformation was carried out and the ratio (LF/HF) of the signal under $0.6 \mathrm{~Hz}(\mathrm{LF})$ and over $0.6 \mathrm{~Hz}$ (HF) was calculated. The component over $\mathrm{LF} / \mathrm{HF}=0.1$ was deleted as an effect of the wire. Finally, the waveforms were reconstituted from the component of the remainder. Then, the 


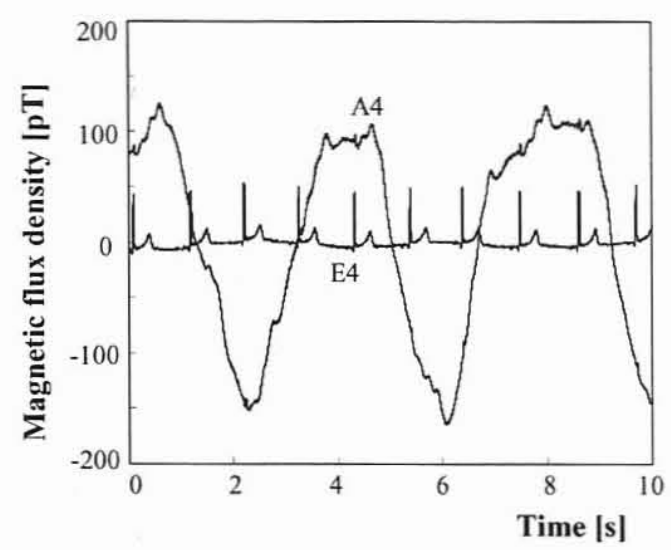

Fig. 2. MCG waveforms with the wire attached for $10 \mathrm{~s}$. A4 is the waveform of the greatest noise, and E4 is the waveform of the smallest noise.

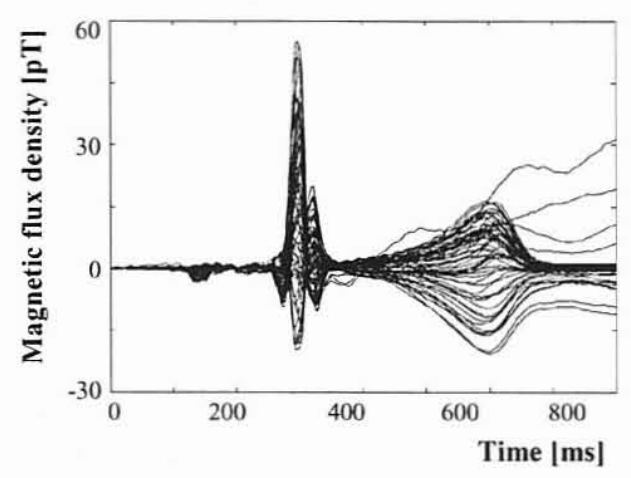

Fig. 3. Averaged MCG waveforms with the wire attached (only average).

MCG waveforms were averaged 40 times in the R wave synchronization of the ECG.

The reasons that data was separated into seven components by PCA and the ratio of $\mathrm{LF} / \mathrm{HF}=0.1$ were experientially decided from the experimental results of five normal subjects. The correlation coefficients of all results were improved.

\section{RESUlTS}

Fig. 2 shows the MCG waveforms with the wire attached for 10 seconds. In Fig. 2, A4 is measurement position of most big noise, and E4 is measurement position of small noise. The noise of the wire appears only in the upper three rows (A1-C8).

Fig. 3 shows the averaged MCG waveforms with the wire attached. The effect of magnetic noise from the wire appears at a $\mathrm{T}$ wave, and it is clear that noise rejection is difficult using the averaging method only.

The MCG waveform is separated into seven components by independent component analysis. The magnetic noise appears in the first, fourth, sixth, and seventh components. Fig. 4 shows the reconstructed MCG waveforms that removed the first, fourth, sixth, and seventh components. The effect of the wire which has appeared the $\mathrm{T}$ wave can be reduced.

For comparison, Fig. 5 shows the averaged MCG waveforms without the wire. The waveforms of Figs. 4 and 5 resemble each other well.

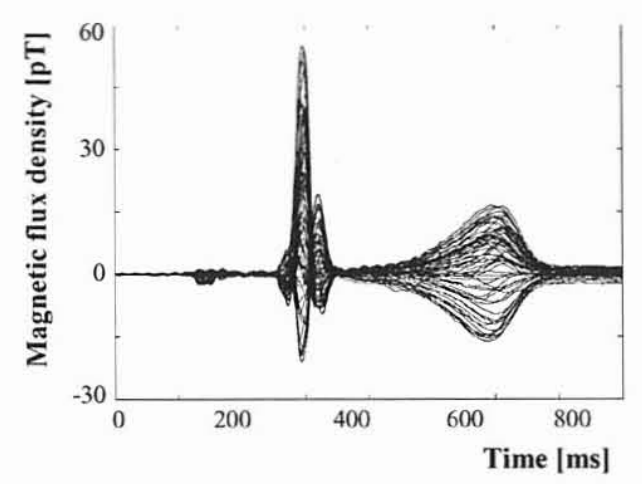

Fig. 4. Reconstructed MCG waveforms that removed the first, fourth, sixth, and seventh components by ICA.

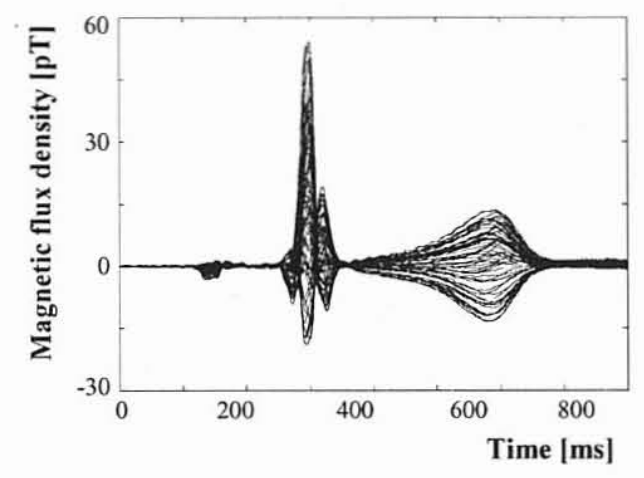

Fig. 5. Averaged MCG waveforms without the wire (no wire).

\section{DISCUSSION}

In order to evaluate this signal processing, the correlation coefficient between the waveforms after signal processing and the waveforms without the wire is calculated. The calculation of the correlation coefficient was divided into upper three rows and bottom five rows.

The correlation coefficients of averaged waveforms (Fig. 3) are 0.839 (upper) and 0.984 (bottom). The correlation coefficients of ICA waveforms (Fig. 4) are 0.941 (upper) and 0.931 (bottom). The correlation coefficient of upper three rows increased, but that of bottom five rows decreased. Because the noise of the wire appears only in the upper three rows. Therefore, upper three rows used the result of ICA, and bottom five rows should use original data. As the result, the correlation coefficient becomes 0.968 and high value. In four MCG waveforms in Fig. 4 are different from that of Fig. 5. Only these MCG waveforms, the correlation coefficients became under 0.9 (A4:0.750, A3:0.781, B4:0.820, B3:0.893). This is the reason that the MCG waveform with the wire has great noise and the correlation coefficients are low (A4 : $-0.174, \mathrm{~A} 3: 0.476, \mathrm{~B} 4: 0.022$, B3 : $0.746)$. It is not possible to remove even in this proposal method. However, it has been greatly improved by ICA.

For the comparison, the result of using digital filter (HPF) is shown at Figs. 6-8. The cut-off frequency of HPF is, respectively, made to be $0.5 \mathrm{~Hz}$ and $1 \mathrm{~Hz}$ and $2 \mathrm{~Hz}$. In Fig. $6(0.5 \mathrm{~Hz}$ HPF), the large noise of the wire appears after a $\mathrm{T}$ wave, and the noise rejection is not sufficient. The correlation coefficient 


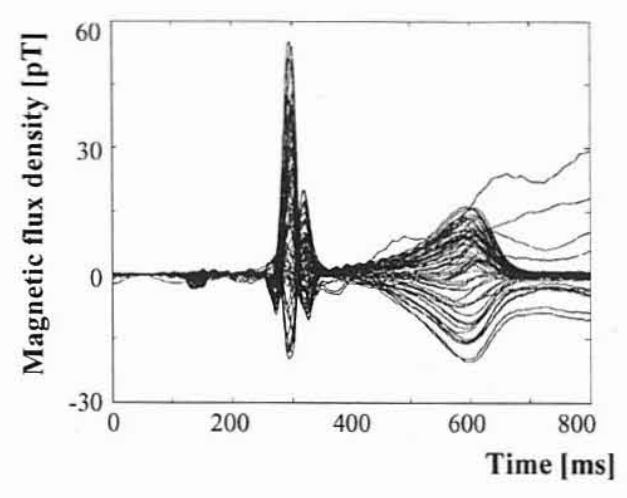

Fig. 6. MCG waveforms with high-pass filter $(0.5 \mathrm{~Hz})$.

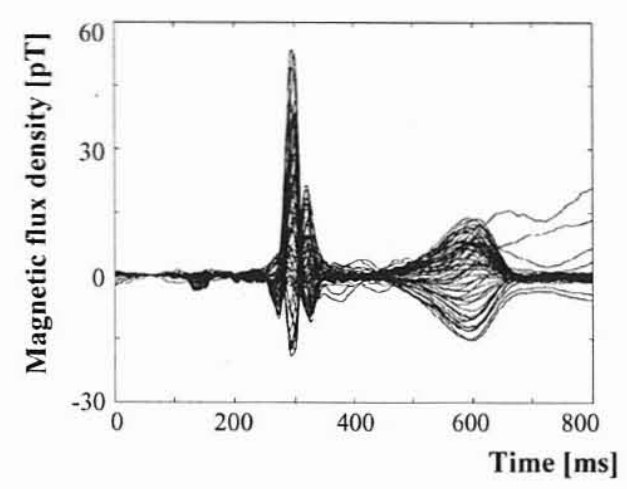

Fig. 7. MCG waveforms with high-pass filter $(1 \mathrm{~Hz})$.

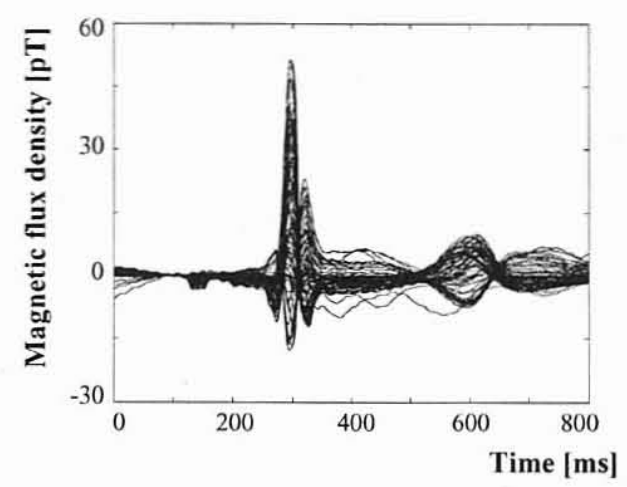

Fig. 8. MCG waveforms with high-pass filter $(2 \mathrm{~Hz})$.

of waveforms is 0.931 . In Fig. 7 ( $1 \mathrm{~Hz} \mathrm{HPF})$, though the noise of the wire decreases, it is not sufficient. Moreover, the peak of $\mathrm{T}$ wave attenuates a little (some $\mathrm{pT}$ ). The correlation coefficient of waveforms is 0.934 . In Fig. 8 ( $2 \mathrm{~Hz}$ HPF), the noise of the T wave decreases, but the peak of $\mathrm{T}$ wave attenuates a large (about $10 \mathrm{pT}$ ). Moreover, the waveform has been distorted. The correlation coefficient of waveforms is 0.896 .

Singer value decomposition (SVD) analysis is carried out MCG with the wire. Noise is removed by same criterion of ICA

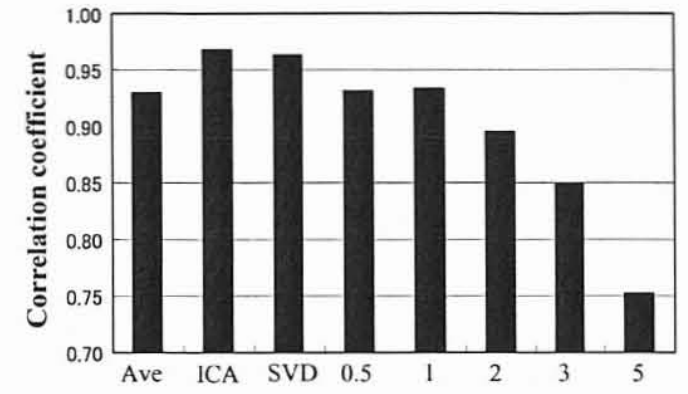

Fig. 9. Correlation coefficients of averaged, ICA, SVD, and high-pass filter $(0.5,1,2,3,5)$ waveforms.

$(\mathrm{LF} / \mathrm{HF}=0.1)$. The correlation coefficient of waveforms is 0.963 and high value.

Fig. 9 shows the correlation coefficients of averaged, ICA, SVD, and HPF $(0.5,1,2,3,5)$ waveforms. The result of ICA is the best the correlation coefficient. ICA is effectively able to carry out the noise rejection in being difficult in digital filter.

Moreover, MCG with two wires attached $(3 \mathrm{~cm}$ and $6 \mathrm{~cm}$ under the episternum) is analyzed by ICA. The correlation coefficient between the waveforms after signal processing and the waveforms without the wire is 0.968 and high value. This proposal method is efficient two wires attached.

\section{CONCLUSION}

Signal processing by ICA, SVD, and digital high-pass filter were carried out in order to reduce the effect of magnetic noise from the used to suture the sternum after heart surgery. In order to evaluate this signal processing, the correlation coefficient was calculated. ICA was the best the correlation coefficient. It was shown that ICA was effective for the removal of the magnetic noise from the wire.

\section{REFERENCES}

[1] J. Nenonen, T. Katila, M. Leinio, J. Montone, M. Makijarvi, and P. Siltanen, "Magnotocardiographic functional localization using current multipole models," IEEE Trans. Biomed. Eng., vol. 38, no. 7, pp. 648-657, Jul. 1991.

[2] K. Kobayashi, Y. Uchikawa, K. Nakai, and M. Yoshizawa, "Visualization of the current-density distribution for MCG with WPW syndrome patients using independent component analysis," IEEE Trans. Magn., vol. 40, no. 4, pp. 2970-2972, Jul. 2004.

[3] P. Takala, H. Hanninen, J. Montonen, M. Makijarvi, J. Nenonen, L. Toivonen, and T. Katila, "Beat-to-beat analysis method for magnetocardiographic recordings during interventions," Phys. Med. Biol., vol. 46, pp. 975-982, 2001.

[4] K. Tsukada, T. Miyashita, A. Kandori, T. Mitsui, Y. Terada, M. Sato, J. Shiono, H. Horigome, S. Yamada, and I. Yamaguchi, "An iso-integral mapping technique using magnetocardiogram and its possible use for diagnosis of ischemic heart disease," Int. J. Cardiac Imaging, vol. 16, pp. 55-66, 2000.

[5] J. F. Cardoso and B. Laheld, "Equivariant adaptive source separation," IEEE Trans. Signal Process., vol. 44, pp. 3017-3030, 1996.

[6] S. Amari and N. Murata, "Independent Component Analysis," Science Corp., Tokyo, Japan, pt. 1, 2002.

Manuscript received February 7, 2005. 